\title{
Repair of ruptured coronary sinus by sole apposition of self-adhesive sealing hemostatic patch
}

\author{
Luca Weltert, MD, ${ }^{a}$ Lorenzo Guerrieri Wolf, MD, ${ }^{a}$ Franco Turani, MD, ${ }^{b}$ and Ruggero De Paulis, MD, ${ }^{a}$ \\ Rome, Italy
}

\author{
From the ${ }^{\mathrm{a}}$ Divisione di Cardiochirurgia, European Hospital, Rome, Italy; and ${ }^{\mathrm{b}}$ Anesthesia and Intensive Care \\ Unit, Aurelia Hospital, Rome, Italy. \\ No sources of funding were received for this work. \\ Disclosures: Authors have nothing to disclose with regard to commercial support. \\ Received for publication March 29, 2018; revisions received July 4, 2018; accepted for publication July 11, 2018; \\ available ahead of print Aug 31, 2018. \\ Address for reprints: Luca Weltert, MD, Divisione di Cardiochirurgia, European Hospital, Via Portuense 700, \\ Rome, Italy (E-mail: 1weltert@gmail.com). \\ J Thorac Cardiovasc Surg 2019;157:e189-91 \\ $0022-5223 / \$ 36.00$ \\ Copyright (C) 2018 by The American Association for Thoracic Surgery \\ https://doi.org/10.1016/j.jtcvs.2018.07.046
}

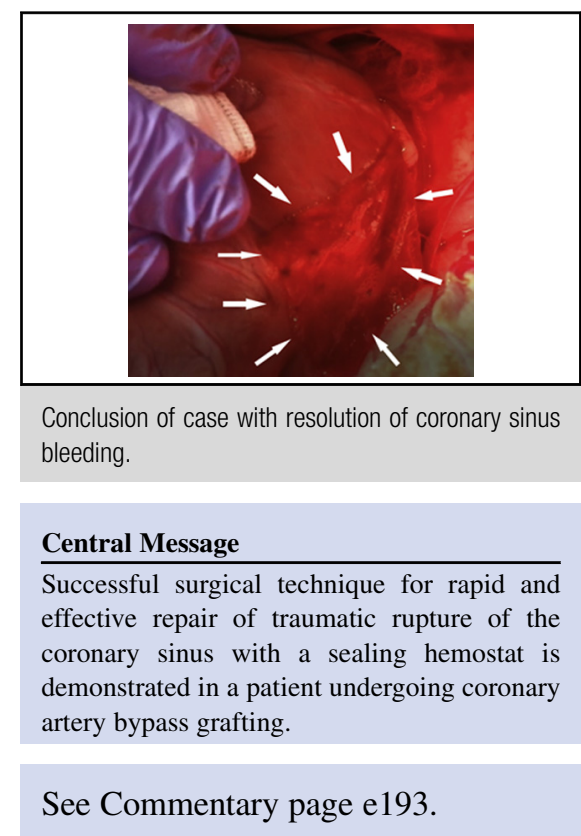

Traumatic rupture of the coronary sinus is an infrequent but well-documented complication of retrograde coronary perfusion during heart surgery procedures. In a retrospective analysis of 1500 patients, preoperative variables of female sex, ejection fraction less than $35 \%$, congestive heart failure, creatinine level greater than $1.5 \mathrm{mg} / \mathrm{dL}$, chronic obstructive pulmonary disease, and lower body mass index were significantly $(P<.05)$ more predominant in those who had coronary sinus rupture occur than in those who did not. In multiple regression and odds ratio (OR) analysis, the factors of female sex (OR, 4.24), age (OR, 1.04), weight (OR, 0.96), and body mass index (OR, 2.10) showed a significant correlation with coronary sinus rupture. ${ }^{1}$ Others have noted that blunt chest trauma, anatomic variants, trauma from cannula insertion, and tissue fragility are also associated with higher risk for coronary sinus rupture. ${ }^{2-6}$ Further, because positioning maneuver is blind, it is also likely to cause occasional iatrogenic damage. Those who had coronary sinus rupture require significantly $(P<.05)$ longer pump times, require longer clamp times, have higher rates of pulmonary complications and infection, and are at higher risk for death. ${ }^{1}$

Rupture of the coronary sinus results in extensive and excessive hemorrhage and blood loss. This usually appears only after removal of the perfusion cannula and after cardiopulmonary bypass weaning. Unfortunately, the
Video clip is available online.

treatment of these types of situations is not standardized, and clinical decision making is complicated by the patient being off the pump and still heparinized.

Very recently (March 9, 2018), we faced a traumatic rupture of the coronary sinus complication in an 84-yearold woman with a body mass index of 18 who was undergoing quadruple coronary artery bypass grafting for a left main coronary artery critical lesion and multiple segmentary lesions (Figure 1). Clinically, the patient had normal anatomy. The procedure involved the use of an autoinflating catheter, and it appeared that the injury occurred during the placement of the device early in the procedure, possibly as a result of the presence of the stiff stylet, which was subsequently retracted. During the procedure, the balloon prevented blood loss; on its removal, however, the rupture became apparent. In an ultimately successful attempt to repair the coronary sinus rupture, we applied a medium-sized sealing hemostatic patch (HEMOPATCH Sealing Hemostat; Baxter Healthcare, Deerfield, Ill).

Our technique consisted of gently lifting the heart without restarting the pump, placing the hemostatic patch, and applying gentle pressure for 3 minutes on the coronary sinus with a dry gauze pad (Figure 2). We bent the dry gauze 


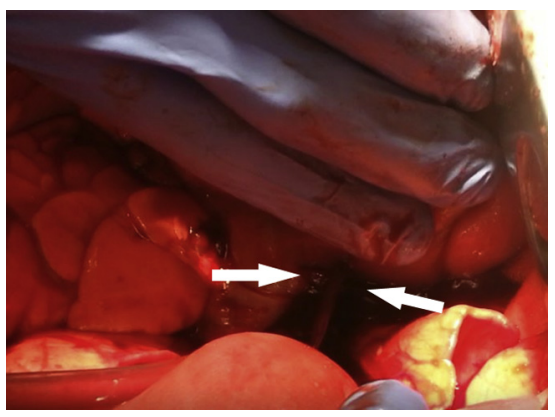

FIGURE 1. Ruptured coronary sinus with hemorrhage. The breach can be appreciated just below the index finger of the operator, as evidenced by the arrow.

pad to create an application pad that was approximately a few millimeters smaller than the sealing hemostatic patch. This sizing of the dry gauze pad allowed its removal after identification of the margin of the sealing hemostatic patch on the heart. The smaller size of the dry gauze pad also minimized the risk of accidental removal of the hemostatic patch. Inspection revealed good positioning of the sealing hemostatic patch, which turned from white and blue to dark red. In an attempt to lift an angle of the hemostatic patch with forceps, there was a firm resistance that confirmed an appropriate "gluing" of the sealing hemostatic patch to the coronary sinus (Figure 3). The bleeding stopped within 3 minutes, full protamine reversal of heparin and decannulation were carried out as normal, and no further treatment was required. Overall, the patient had minimal blood loss (350 mL during the first 24 postoperative hours) and was weaned from the ventilation approximately 7 hours after sternal closure. The remainder of her postoperative course was normal, and she was discharged to the cardiac rehabilitation service. On routine echocardiographic evaluation, the sealing hemostatic patch was

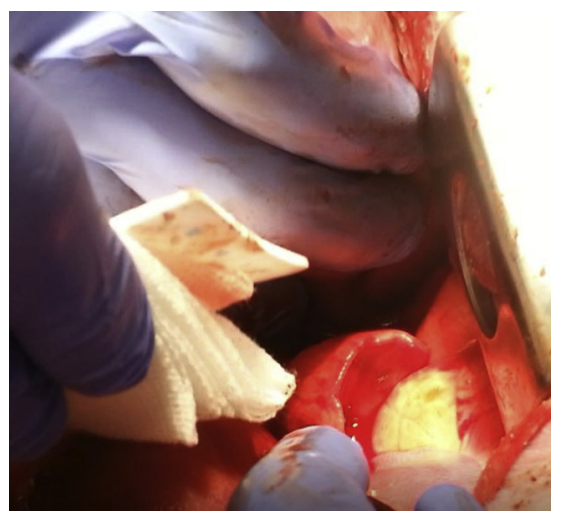

FIGURE 2. Surgeon positioning of sealing hemostatic patch. The gauze is plicated, leaving a few millimeters of free margin for the sealing hemostatic patch, which facilitates retrieval of the gauze without accidental removal of the patch itself.

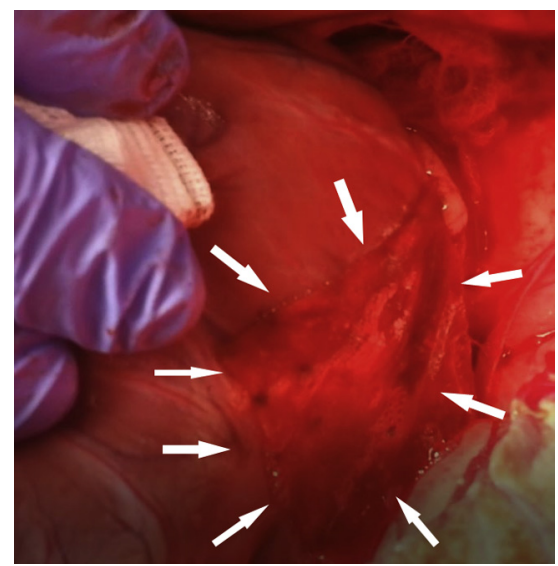

FIGURE 3. Conclusion of case with resolution of coronary sinus bleeding. The hemostatic sealing patch contour (arrows) can barely be distinguished. Some of the blue squares are still visible and help in identifying the patched zone. No bleeding is present, and no distortion of the normal anatomy is induced.

undetectable, and no significant pericardial effusion was detected. Video 1 illustrates the procedure and outcome of the ruptured coronary sinus and its repair.

We established the feasibility of this technique and its execution in animal studies (heparinized and nonheparinized piglets) performed at Baxter Healthcare Facilities. To our knowledge, however, this is the first documented case of this sealing hemostatic patch being used for the cessation of traumatic rupture and excessive bleeding of the coronary sinus in a human surgical patient. In summary, the fast (within 3 minutes), safe, and efficient single

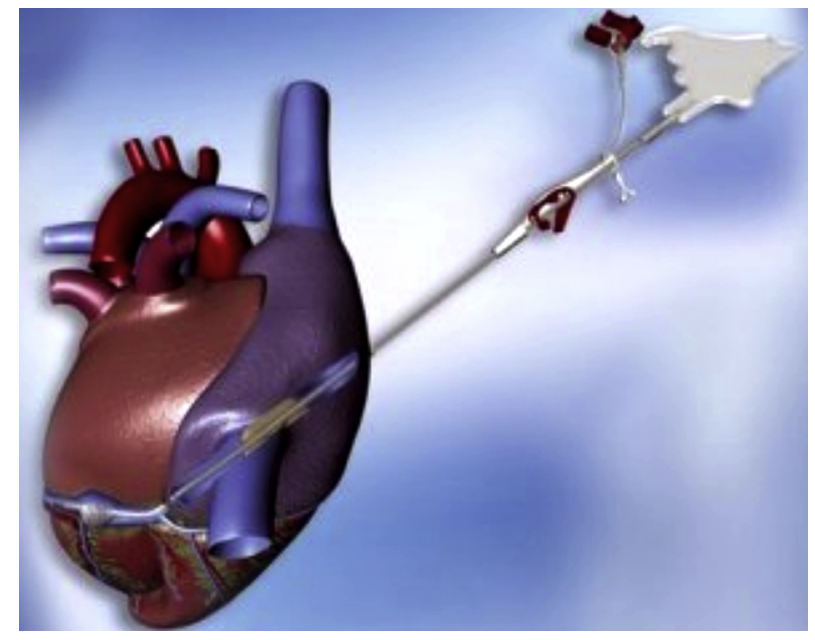

VIDEO 1. As seen in this video clip, insertion of the catheter and its placement in the coronary sinus through the right atrium may result in a traumatic ruptured coronary sinus. Our technique, which uses a hemostatic sealing patch and a dry gauze pad, results in hemostasis and a successful repair of this ruptured coronary sinus. Video available at: https://www. jtcvs.org/article/S0022-5223(18)32036-1/fulltext. 
application of a sealing hemostatic patch is a significant beneficial therapeutic treatment strategy for surgeons who have patients with the high-risk complication of coronary sinus rupture.

\section{References}

1. Sabzi F, Zokaei A. Factors predicting coronary sinus rupture following cannula insertion for retrograde cardioplegia. Clin Med Insights Cardiol. 2012; 6:1-6.
2. Kim DW, Lee KS, Na KJ, Oh SG, Jung YH, Jeong IS. Traumatic rupture of the coronary sinus following blunt chest trauma: a case report. J Cardiothorac Surg. 2014;9:164.

3. Kurusz M, Girouard MK, Brown PS Jr. Coronary sinus rupture with retrograde cardioplegia. Perfusion. 2002;17:77-80.

4. Kshettry VR, Salerno CT, Lakhanpal S, Kroshus T. Coronary sinus injury due to retrograde cardioplegia: a report of three cases. J Card Surg. 1996;11:359-62.

5. Bergman RA, Thomson SA, Saddeh FA. Absence of the coronary sinus. Anta Anz. 1988;166:9-12.

6. Ortale JR, Grill EH. Case of bilateral vena cava with variation in the azygos system and in the heart. Ann Anat. 1994;176:323-5. 